\title{
Clinical Practice and Evidence-based Knowledge: Reducing Urinary Tract Infection in Elderly Hip Fracture patients
}

\author{
Liv Wergeland Sørbye ${ }^{1 *}$, Mette Irene Martinsen², Else Vengnes Grue ${ }^{3}$ \\ ${ }^{1}$ VID Specialized University, Institute of nursing and health, Box 124, Vinderen, 0319 Oslo, Norway \\ ${ }^{2}$ Department of surgery, Quality coordinator Diakonhjemmet Hospital, Diakonveien 12, 0370 Oslo, Norway \\ ${ }^{3}$ Emerita assistant professor, VID Specialized University, Institute of nursing and health, Box 124, Vinderen, 0319 Oslo, Norway
}

Received: July 26, 2016; Accepted: : July 28, 2016; Published: August 06, 2016

*Corresponding author: Liv Wergeland Sørbye, VID Specialized University, Institute of nursing and health, Box 124, Vinderen, 0319 Oslo, Norway, E-mail: liv.wergeland.sorbye@vid.no

\begin{abstract}
Objective: Urinary Tract Infections (UTIs) are a common complication of hip fracture, but quality assurance processes might help reduce their frequency. In this study, we measured the effect of systematic quality prevention UTI with hip fracture.

Materials and Methods: Our analyses involved three samples: A, $\mathrm{n}=331$, pre-intervention (2004-2006); B, $\mathrm{n}=319$ (2013-2014), post-intervention one; and C, $n=349$ (May 2015-March 2016), postintervention two. Inclusion criteria were aged $\geq 65$ years, hip fracture and admission from home to acute care hospitals. From 2012, the hospital participated in a national patient safety program to prevent UTIs, emphasizing indications for indwelling urinary catheters (IUCs). Education and practice for sterile catheter insertion and removal the first morning after surgery. In 2015, a daily risk-assessment meeting with the staff was implemented. One focus was following up on UTI and the use of IUC.

Results: Samples A and B did not differ for UTI rates, but A and $C$ did $(12.7 \%$ vs $7.2 \% ; p=0.02)$ and $B$ and $C(17.9 \%$ vs $7.2 \%$; $<$ 0.01). Samples $A$ and $C$ had a significant correlation of length of stay (LOS) and UTI compared to no UTI (A, p $<0.001$ and $\mathrm{C}, \mathrm{p}=0.002)$ while sample $B$, was close to significant $(p=0.057)$. The median LOS decreased from 11 days in sample A to 6 days in samples $B$ and $C(p=$ $0.01)$ due to a governmental coordination reform. Logistic regression revealed three significant predictors for UTI $(p=0.000)$ : age $>81$ year, first intervention group (Sample B), LOS > 11 days.

Conclusion: Staff quality assurance training requires expertise, time, and engagement to facilitate reduction in UTIs among hip fracture patients. Quality improvement takes time, commitment and continues follow up.
\end{abstract}

Keywords: Hip fracture; Urinary tract infection; Intervention; Safety program

\section{Abbreviation}

UTI: Urinary Tract Infection; IUC: Indwelling Urinary Catheter; LOS: Length Of Stay

\section{Introduction}

Hip fracture is a common trauma among the old and frail and is associated with complications, decline in function and mobility, and a high mortality rate. Urinary Tract Infection (UTI) is one of the most common complications among hip fracture patients and is linked to prolonged Length Of Stay (LOS) and increased delirium incidence [1] Use of an indwelling catheter is a great risk factor for UTI [2]. Many hip fracture patients experience urinary retention pre-operatively, and an indwelling urinary catheter (IUC) is often inserted before surgery. Several authors have discussed the use of IUCs during surgery [3-5], arguing the pros and cons. As is widely known, the time span from inserting the catheter to its removal has a critical effect on UTI frequency in the hospital setting [6-7].

In addition to number of days with IUC, the hygiene and sterile procedures of the staff are important. For these reasons, staff should be familiar with the International Guidelines to Prevent UTI [8-9]. In practice, the staff needs to focus on IUC and unnecessary use.

In this work, we present a follow-up study on an earlier report [5]. Here we describe an intervention based on a national patient safety program. The aim was to evaluate the effect of systematic quality prevention of UTI. The research questions were as follows: How can UTIs be reduced in older hip fracture patients, and what are the success-related factors in reducing UTI in these patients?

\section{Materials and methods}

The study population consisted of three groups of hip fracture patients admitted to an Orthogeriatrics unit at Diakonhjemmet Hospital in three time periods. The unit covers a population of approximately 80,000 (13\%) inhabitants aged 65 years or older in Oslo, Norway [10]. Inclusion criteria were hip fracture patients aged 65 years or older, living in their own home. Sample 
A (2004-2006) was pre-intervention and formed the basis for a secondary analysis of a dataset from previous work [5]. Sample B (2013-2014) consisted of patients treated after a first staff intervention, and sample C(May 2015-March 2016) was after a second intervention. In A, we used the Resident Assessment Instrument for Acute Care [11] for collecting quantitative data. This instrument aids in assessing the situation of the older patient in an acute care setting [12]. Samples B and C were based on routine data from a local quality registry. The records included demographic, medical, and functional data collected from routine assessment by the interdisciplinary team for quality-improvement purposes. In all of the three samples, UTI had an ICD-10 code N39.0 in the electronically journal. Criteria for medical treating of UTI were a combination of different clinical symptoms: frequent urination, strong odor, pain when voiding, retention, fever and signs of delirium (often seen on day 2 or 3 after surgery). The urine was tested with a dipstick and treatment started on indication of bacteriuria. The urine was sent to cultivation. A culture more than $10.000 \mathrm{CFU} / \mathrm{ml}$ confirmed the diagnosis.

The second author was responsible for the updating and control of the local register data. The third author was responsible for the data in sample A. They had access to the electronical journal for retrospective control of their data. All hip fracture patients 65 years or older were included. The hospital had access to the national death register, and date of death was retrospectively added to the local register data. For sample $\mathrm{C}$, this data is not yet transferred.

\section{Ethical consideration}

Ethical approval was obtained for all samples according to national regulations. Regional Committees for Medical and Health Research Ethics approved sample A (ref. no. 186-04032 and 2011/1322). The Privacy Ombudsman for Research and the hospital research board approved the quality registry.

\section{The first intervention}

The guidelines before the first intervention were that all patients received an IUC before surgery and that the IUC was to be removed on the first morning after surgery. The practical application of these guidelines was that the nurses inserted an IUC shortly after admission and did not always remove it before day 2 or 3 after surgery. When implementing the safety program in 2012, the project leaders revised and updated all guidelines concerning urinary catheters. The unit especially focused on indications for use of IUC versus intermittent catheterization. A physician was to be the one to give an order for IUC insertion. In addition, one of the senior nurses taught the nursing staff about the new guidelines and procedures for IUC insertion.

The protocol was that on the first morning after surgery, the night shift staff should remove IUCs. The nurses and physicians were to evaluate daily the indication for eventual further need for IUC. The staff assessed patients with symptoms of delirium such as being easily distracted and having episodes of disorganized speech, variable mental function over the course of the day, or acute change in mental status from baseline, all possible UTI symptoms. The staff registered each incidence of UTI on a calendar, and all patients with a UTI received appropriate observation and treatment.

\section{The second intervention}

In 2015 , the team leader decided to implement daily riskassessment meetings with the nurses. One focus was following up on IUC use and UTIs. To provide better patient monitoring, the unit leader introduced a whiteboard on the floor. Every weekday, the nurses gathered and discussed whether the patients had an indication for IUC, not insert an IUC before close to surgery time and removal by the night shift on the first morning after surgery and focusing on criteria for UTI, such as retention, frequent urination, strong odor, pain when voiding, fever, and delirium. If the patient was diagnosed with UTI, this diagnosis was marked on the white board.

\section{Statistics}

We present continuous variables as means, ranges and Standard Deviations (SDs), medians, and categorical variables as numbers and percentages. The frequency of LOS age was divided in three groups based on frequency (Table 3). Chi-square tests were used to compare dichotomous variables between two groups, independent t-tests to compare mean age and non-parametric tests to compare LOS. Conditions significantly associated with UTI $(\mathrm{P}<0.05)$ were entered into a forward logistic regression model, with UTI as the dependent variable. Results from both the cross tables and the regression model are reported as Odds Ratios (OR) with 95\% Confidence Intervals (CI). Data were analyzed with SPSS software version 23.0 (SPSS Inc., Chicago, IL).

\section{Results and Discussion}

The study included 999 patients divided into three samples over a time span of 12 years. Each group had the same mean age 84 years, (65-102), and 20-28\% was male. (Table 1) compares the outcomes between the samples. The UTI frequencies were $13 \%$ and $18 \%$ in $A$ and B, respectively but $7.2 \%$ in C, a significant reduction compared to the other two groups ( $p=0.001$ ). Comparing samples B and C did differ in UTI rates, (17.9\% vs 7.2 $\% ; \mathrm{p}<0.01$ ). This result suggests that the second intervention with daily follow-up had an effect.

The introduction of governmental coordination reform required decreasing LOS in the hospital unit [13]. An unexpected result in this study was a decrease in the median LOS from 11 days in A to 6 days in B and C, respectively, which was quite dramatic by Norwegian standards. An Australian study documented a LOS of 35 days for community-dwelling patients [14].

The pre- intervention group seems for us appropriate for comparison due to the major reorganization that took place with a great impact on LOS. In sample "A" we had the lowest rate of UTI. We had expected a lower rate of UTI due to a shorter LOS in sample B and C. 


\begin{tabular}{|c|c|c|c|c|c|}
\hline Characteristics & $\mathrm{n}=331(\mathrm{~A})$ & $n=319(B)$ & $n=349(C)$ & $\begin{array}{l}P \text { value } \\
A \text { and } B\end{array}$ & $\begin{array}{l}P \text { value } \\
A \text { and } C\end{array}$ \\
\hline $\begin{array}{l}\text { Gender, n (\%): } \\
\text { Male } \\
\text { Female }\end{array}$ & $\begin{array}{l}67(20.2) \\
264(79.8)\end{array}$ & $\begin{array}{l}91(28.5) \\
228(71.5)\end{array}$ & $\begin{array}{l}87(24.9) \\
262(75.1)\end{array}$ & 0.017 & 0.169 \\
\hline Age, mean (range) (SD) & $84.3(65-100)(6.7)$ & $84.6(65-100)(7.9)$ & $84.0(65-102)(8.2)$ & 0.574 & 0.585 \\
\hline Urinary tract infection, $\mathbf{n}(\%)$ & $42(12.7)$ & $57(17.9)$ & $25(7.2)$ & 0.080 & 0.020 \\
\hline Delirium, n (\%) & $50(15.1)$ & $99(31.0)$ & $59(17.0)$ & 0.001 & 0.531 \\
\hline${ }^{a}$ LOS in days, median (range) (SD) & $11(2-110)(10.3)$ & $6(1-80)(5.2)$ & $6(1-24)(2.7)$ & 0.001 & 0.001 \\
\hline${ }^{\mathrm{b}}$ Death after one-year n (\%) & $52(15.7)$ & $79(24.8)$ & & 0.04 & \\
\hline
\end{tabular}

Table 2: Characteristics of patients with UTI and those with no-UTI among the three samples.

\begin{tabular}{|c|c|c|c|c|c|c|c|c|c|}
\hline \multirow{2}{*}{ Characteristics } & \multicolumn{2}{|c|}{$n=331(A)$} & \multicolumn{4}{|c|}{$n=319(B)$} & \multicolumn{3}{|c|}{$n=349(C)$} \\
\hline & UTI & Not UTI & $P$ value & UTI & Not UTI & P value & UTI & Not UTI & P value \\
\hline $\begin{array}{l}\text { Gender, n (\%) } \\
\text { Male Female }\end{array}$ & $\begin{array}{l}8(19.0) \\
34(81.0)\end{array}$ & $\begin{array}{l}59(20.4) \\
230(79.6)\end{array}$ & 1.0 & $\begin{array}{l}11(19.3) \\
46(80.7)\end{array}$ & $\begin{array}{l}80(30.5) \\
182(69.5)\end{array}$ & 0.106 & $\begin{array}{l}7(28.0) \\
18(72.0)\end{array}$ & $\begin{array}{l}80(24.7) \\
244(75.3)\end{array}$ & 0.810 \\
\hline Age, mean (SD) & 87.1(6.4) & 83.4 (6.7) & 0.003 & $86.7(8.9)$ & 84.1(8.0) & 0.028 & $84.7(8.0)$ & $83.9(8.2)$ & 0.627 \\
\hline Delirium, n (\%) & $9(21.4)$ & $41(14.2)$ & 0.248 & $23(40.4)$ & $76(29.0)$ & 0.114 & $5(20.0)$ & 54 (16.7) & 0.590 \\
\hline $\begin{array}{l}\text { LOSa in days, median } \\
\text { (SD) }\end{array}$ & 15 (18.2) & $10(8.1)$ & 0.001 & 7 (3.7) & $6(5.4)$ & 0.057 & $7(4.3)$ & $6(2.5)$ & 0.002 \\
\hline Death after one year & $8(15.4)$ & $44(84.6)$ & 0.87 & 13(16.5) & $66(83.5)$ & 0.87 & - & - & - \\
\hline
\end{tabular}

The reduction identified here may have consequences. Survival rates for one-year follow-up studies after hip fracture vary from $14 \%$ to $36 \%$ [15]. The current study showed a significant increase in death rates $(\mathrm{p}<0.05)$ from $15.7 \%$ for $\mathrm{A}$ to $26.7 \%$ for B. Patients who were critically ill were excluded in sample A this may be a part of the explanation for lower death rate. A Swedish study show, however, that a shorter hospital stays increased mortality odds [16], so we might assume that reduced LOS have some influence on the higher death rate. Although LOS was reduced in samples "B" and "C" patients with UTI had a statistically significantly longer LOS $(p<0.05)$ than those without UTI, in agreement with an earlier report [15]. We do not have data about one- year-mortality for Group C. We found differences in one-year mortality between $A$ and $B(p=0.04)$. Our data showed no connection between one-year mortality and UTI, as earlier documented [17]. In the current work, we found no statistically significant correlation between UTI and delirium as others have reported [1]. One reason for the discrepancy could be that older patients with a hip fracture may have symptoms of delirium independent of a UTI.

The logistic regression revealed that belonging to the first intervention group, sample B, gave an increased risk for UTI;
OR $2.58(\mathrm{CI}=1.70-3.91)$. This period from 2012 to 2014 was a time of changing with new routines and workloads, due to the coordination reform [13]. Two other predictors remaining in the final stepwise model age $>81$ year, $\mathrm{OR}=0.53(\mathrm{CI}=0.33-0.84)$ and LOS $>11$ days $2.61(\mathrm{CI}=1.67-4.10)$. Younger older is more fit, and is less at risk for UTI [18]. To get an UTI in a hospital setting, may reduce the total health situation and prolong the LOS. The hip fracture patient could acquire other complication while in hospital and be more exposed for infection.

A national program in US hospitals targeting reduced rates of catheter-associated UTI has found that a collaborative effort focusing on both technical and socio adaptive interventions could reduce rates in non-intensive care units but that intensive care units saw no decreases [9]. Catheters are a main factor in healthcare-associated infections in hospitals, and the most important preventive measure is reduced use [6-8], yet it seems difficult to use alternatives to IUCs.

Quality assurance processes offer one possible avenue. For example, one study showed [5] that during treatment of patients in the current sample A, the staff knew that IUCs should be removed 24 hours after hip fracture surgery. Despite this understanding, however, at 72 hours, 35 (11\%) still had the IUC in place. 
The first intervention in this study, with training in guidelines and procedures, had little effect on the use of IUC and UTI. One reason may be that only one nurse in the unit was responsible for implementing the program, with support from staff. The leader team on the ward was only involved to a small degree with the implementation and program follow-up, and followup therefore was limited. This limitation might explain why we found no UTI reduction in sample B: Leadership and continuous follow-up are important when changing practice, and our study shows an apparent beneficial effect when the team leader held daily meetings and presented results to the nurses (second intervention). However, we cannot state with certainty that a reduction in the use of IUC was the sole factor in reduced UTIs because there was a simultaneous focus on treating patients with other symptoms than just bacteriuria. The effects might thus be from a combination of factors.

Ward meetings for risk reduction are a cost-effective and transparent way to work. In Diakonhjemmet Hospital, they are associated with more tangible improvements, ensuring systematic measurement of risk areas and regular meetings to discuss incidents. The patient safety culture has improved through daily discussions about risk areas, and measures to evaluate patients at risk have been more easily implemented because they are requested and discussed in the meetings.

Improvement does not happen behind a desk but takes place "on the floor". For this reason, it is important to create possible new forms and tools for floor use and to discuss not only the risks but also the actions associated with risk and with reducing it Employee involvement in the process is critical, and the process search should focus on advantages, not perfection.

Hip fracture patients often experience urinary retention preoperatively, are dehydrated or have urinary incontinence. These problems in addition to delayed surgery as well as hygienic reasons on theater may cause insertion of IUC preoperatively. An important reason for not removing the IUC after surgery was low urine production, and this condition had to be monitored. Also, IUC reduced the necessity need help to get to the toilet.

The hospital really wanted to reduce UTI and in Group C staff started to scan the bladder, performed intermittent catheterization on admission for all patients, and thereby delayed the use of IUC. At the Daily risk meetings, they focused on removing the IUC and administer fluid per OS or intravenous to stimulate urine output. These initiatives may have had an impact on the positive result.

The second question for any proposed change or process could be, why do we measure this? In this intervention study, all of the patients had an IUC during the surgery. However, after several years of focus on reducing the time the patients had an IUC, sterile procedures and UTIs, this now seems to be integrated into daily practice at the unit.

\section{Limitations and Strengths}

This study had several limitations. It involved a qualityimprovement project associated with a national safety program.
As a pre-intervention sample, we used secondary analyses of a dataset from 2004-2006. This study was concern about vision and hearing impairment in patient with hip fracture. However, in this study with older people $65+$, only $2 \%$ was excluded due to intact vision and hearing abilities and $1.6 \%$ assessed as critical ill on the inclusion day [19]. The effect of the two intervention (B and C) was compared to A the pre-intervention sample. The quality registry for patients with hip fracture has to be followed up in addition to the electronic medical records. In this study, we used few variables for measuring the effects of our intervention. However, the material had solid data on age, gender, LOS, "oneyear survival," and using ICD-10 code for hip fracture and UTI.

\section{Conclusion}

Quality assurance training of staff requires expertise and engagement to enable reductions in urinary infections among hip fracture patients. Urinary tract infection is a complication that increase frailty, and might lead to an earlier death. Staff education alone is not enough to reduce UTIs in this patient group. Changes in practice take time, patience, and commitment.

\section{Acknowledgements}

We would like to show our gratitude to the staff at the orthopedically ward in Diakonhjemmet hospital for their support of our clinical research.

\section{Declarations}

The authors certify that there is no conflict of interest with any financial, research, and academic organization, with regards to the research work discussed in the manuscript.

Table 3: Comparing patients with a Urinary Tract Infection (UTI), with those who $\operatorname{did} \operatorname{not} \mathrm{N}=999$.

\begin{tabular}{|c|c|c|c|c|}
\hline $\begin{array}{l}\text { Selected } \\
\text { characteristics }\end{array}$ & $\begin{array}{l}\text { UTI N } \\
\text { (\%) }\end{array}$ & $\begin{array}{c}\text { No UTI N } \\
(\%)\end{array}$ & OR (CI) & $\begin{array}{l}\text { Chi- } \\
\text { square } \\
\text { p-value }\end{array}$ \\
\hline $\begin{array}{l}\text { Age } \\
65-81 \text { vs. } 82 \text { or more }\end{array}$ & $25(20.2)$ & $\begin{array}{c}299 \\
(33.37\end{array}$ & $\begin{array}{l}1.96 \\
09-296)\end{array}$ & 0.003 \\
\hline $\begin{array}{l}82-88 \text { vs } 65-81 \text { and } \\
89-102\end{array}$ & 48 (38.7) & $350(36.0)$ & $\begin{array}{c}(1.29-2.96) \\
1.12(0.76-1.65)\end{array}$ & No sig. \\
\hline $89-102$ vs 88 or less & $51(41.1)$ & 269 (30.7) & $1.57(1.07-2.31$ & 0.02 \\
\hline Delirium & 37 (29.8) & 171 (19.5) & $\begin{array}{c}1.75 \\
(1.15-2.66)\end{array}$ & 0.008 \\
\hline $\begin{array}{l}\text { Length of stay } \\
1-5 \text { days }\end{array}$ & $25(20.2)$ & $288(26.0)$ & $\begin{array}{c}0.71 \\
(0-45-1.14)\end{array}$ & No sig \\
\hline 6-11 days & $58(46.8)$ & $475(54.3)$ & $\begin{array}{c}0.74 \\
(0.51-1.08)\end{array}$ & No sig \\
\hline More than 11 days & $40(32.3)$ & $171(19.5)$ & $2.2(1.5-3.2)$ & 0.001 \\
\hline $\begin{array}{l}\text { Samples } \\
\text { Pre-intervention } \\
(\mathrm{N}=331)\end{array}$ & 42 (33.9) & $289(33.0)$ & $1.04(0.70-1.55)$ & No sig. \\
\hline $\begin{array}{l}\text { First intervention } \\
(\mathrm{N}=319)\end{array}$ & $57(46.0)$ & $262(29.9)$ & $2.0(1.36-2.92)$ & 0.000 \\
\hline $\begin{array}{l}\text { Second intervention } \\
(\mathrm{N}=349)\end{array}$ & $25(20.2)$ & $324(37,0)$ & $0.43(0.27-0.69)$ & 0.001 \\
\hline
\end{tabular}




\section{References}

1. Kamel HK. The frequency and factors linked to a urinary tract infection coding in patients undergoing hip fracture surgery. J Am Med Dir Assoc. 2005;6(5):316-20.doi: 10.1016/j.archger.

2. Beveridge LA, Davey PG, Phillips G, McMurdo ME. Optimal managemen of urinary tract infections in older people. Clinical interventions in aging. 2011;6:173-80. doi: 10.2147/CIA

3. Schwartz BC, Frenette C, Lee TC, Green L, Jayaraman D. Novel lowresource intervention reduces urinary catheter use and associated urinary tract infections: role of outcome measure bias? American journal of infection control. 2015;43(4):348-53. doi: 10.1016/j.ajic.

4. Halleberg Nyman M, Gustafsson M, Langius-Eklof A, Johansson JE, Norlin R, Hagberg L. Intermittent versus indwelling urinary catheterisation in hip surgery patients: a randomised controlled tria with cost-effectiveness analysis. International journal of nursing studies. 2013;50(12):1589-98. doi: 10.1016/j.ijnurstu.

5. Sørbye LW, Grue EV. Hip fracture and urinary incontinence use of indwelling catheter postsurgery. Scandinavian journal of caring sciences. 2013;27(3):632-42. doi: 10.1111/j.14716712.2012.01075.x.

6. Lo E, Nicolle L, Classen D, Arias KM, Podgorny K, Anderson DJ, et al. Strategies to prevent catheter-associated urinary tract infections in acute care hospitals. Infection control and hospital epidemiology. 2008;29 Suppl 1:S41-50. doi: 10.1086/591066.

7. Gould CV, Umscheid CA, Agarwal RK, Kuntz G, Pegues DA. Guideline for prevention of catheter-associated urinary tract infections 2009 Infection control and hospital epidemiology. 2010;31(4):319-26. doi: 10.1086/651091

8. Hooton TM, Bradley SF, Cardenas DD, Colgan R, Geerlings SE, Rice JC, et al. Diagnosis, prevention, and treatment of catheter-associated urinary tract infection in adults: 2009 International Clinical Practice Guidelines from the Infectious Diseases Society of America. Clinical infectious diseases. 2010;50(5):625-63. doi: 10.1086/650482.

9. Saint S, Greene MT, Krein SL, Rogers MA, Ratz D, Fowler KE, et al. A Program to Prevent Catheter-Associated Urinary Tract Infection in Acute Care. N Engl J Med. 2016;374(22):2111-9. doi: 10.1056/ NEJMoa1504906. 1.

10. Oslo kommune Statistikkbanken. 2014. (Municipality of Oslo. Statistics 2014): http://statistikkbanken.oslo.kommune.no/.

11. Carpenter GI, Teare GF, Steel K, Berg K, Murphy K, Bjørnson J, et al. A new assessment for elders admitted to acute care: reliability of the MDS-AC. Aging (Milan, Italy). 2001;13(4):316-30. doi: 10.1007/ BF03353428.

12. Gray LC, Bernabei R, Berg K, Finne-Soveri H, Fries BE, Hirdes JP, et al. Standardizing assessment of elderly people in acute care: the interRAI Acute Care instrument. Journal of the American Geriatrics Society. 2008;56(3):536-41.doi: 10.1111/j.1532-5415.2007.01590.x.

13. Romøren TI, Torjesen DO, Landmark B. Promoting coordination in Norwegian health care. International journal of integrated care. 2011;11: e127.

14. Ireland AW, Kelly PJ, Cumming RG. Total hospital stay for hip fracture: measuring the variations due to pre-fracture residence, rehabilitation, complications and comorbidities. BMC Health Serv Res. 2015;15:17. doi: 10.1186/s12913-015-0697-3.

15. Carpintero P, Caeiro JR, Carpintero R, Morales A, Silva S, Mesa M. Complications of hip fractures: A review. World journal of orthopedics. 2014;5(4):402-11. doi: 10.5312/wjo.v5.i4.402.

16. HallebergNyman M,JohanssonJE,Persson K, Gustafsson M.Aprospective study of nosocomial urinary tract infection in hip fracture patients. Journal of clinical nursing. 2011;20(17-18):2531-9.10.1111/j.13652702.2011.03769.x

17. Nordström P, Gustafson, Michaëlsson K, Nordström A. Length of hospital stay after hip fracture and short term risk of death after discharge: a total cohort study in Sweden. BMJ. 2015;350:h696. doi: 10.1136/bmj.h696

18. Rowe TA, Juthani-Mehta M. Diagnosis and management of urinary tract infection in older adults. Infectious disease clinics of North America. 2014;28(1):75-89.

19. Grue EV, Kirkevold M, Mowinchel P, Ranhoff AH. Sensory impairment in hip-fracture patients 65 years or older and effects of hearing/vision interventions on fall frequency. J Multidiscip Healthc. 2009;2: 1-11. doi:10.2147/JMDH.S4126. 